\title{
MONITOREO DE SALUD ESTRUCTURAL
}

* Estudiante de Ingeniería Civil de la Universidad del Valle.

** Ph.D. Profesor Titular de la Universidad del Valle - Director de la Escuela de Ingeniería Civil y Geomática.

*** Ph.D. Profesor Titular de la Universidad del Valle.

\author{
Johannio Marulanda C. \\ Peter Thomson, Ph. $\mathrm{D}^{\star *}$ \\ Johannio Marulanda A. Ph. ${ }^{\star \star \star}$
}

\section{RESUMEN}

Los métodos no destructivos para detección de daños en estructuras civiles han ganado especial atención en los últimos años por parte de investigadores de todo el mundo, debido a los adelantos en tecnología de instrumentación y procesamiento digital de señales. Las técnicas de Monitoreo de Salud Estructural permiten identificar la presencia, ubicación y magnitud del daño a partir de cambios en sus características estáticas y dinámicas. Estas técnicas se pueden 
implementar a distancia y en tiempo real, lo que reduce costos de inspección y tiempo de alerta. En la Escuela de Ingeniería Civil y Geomática de la Universidad del Valle se está desarrollando un proyecto sobre Monitoreo de Salud Estructural en el puente "El Hormiguero" y se tiene suscrito un convenio con la Universidad Tecnológica de Pereira para el monitoreo del Viaducto Pereira-Dos Quebradas.

\section{ABSTRACT \\ Non-destructive methods for damage detection in civil structures have recently gained the attention of researchers throughout the world due to the advances in instrumentation technology and digital signal processing. Structural Health Monitoring techniques allows the presence, location and magnitude of damage to be detected due to changes in the static and dynamic characteristics of the structure. These techniques can be implemented remotely and in real-time, which can significantly reduce inspection costs and provide an instantaneous alert signal due to damage in the structure. The School of Civil Engineering and Geomatics of the Universidad del Valle has a Structural Health Monitoring research project that includes real- time monitoring of the Hormiguero bridge and an agreement with the Universidad Tecnológica de Pereira for joint monitoring of the Pereira-Dos Quebradas Viaduct.}

\section{INTRODUCCIÓN}

El interés en las técnicas para monitorear una estructura y descubrir un daño en su etapa más temprana, se ha incrementado dentro de las comunidades de ingenieros civiles, mecánicos y aeroespaciales. Los métodos actuales de identificación de daño son visuales o de localización experimental, como los métodos acústicos o ultrasónicos, métodos de campo magnético, radiográficos o los métodos de campo térmico. Todas estas técnicas experimentales requieren que la vecindad del daño sea conocida a priori y que la porción de la estructura a inspeccionar sea accesible. Sujetos a estas limitaciones, estos métodos experimentales pueden detectar el daño en la superficie de la estructura o cerca de ella.

La necesidad de desarrollar técnicas de detección de daños globales que puedan aplicarse a estructuras complejas, ha llevado al desarrollo de métodos que examinan cambios en las características de vibración de la estructura. La idea básica es que los parámetros dinámicos (frecuencias, modos de vibración y de amortiguamiento) cambian en función de las propiedades físicas de la estructura (masa, amortiguamiento y rigidez). Por consiguiente, los cambios en las propiedades físicas causarán cambios en las propiedades dinámicas, abriendo un camino para la localización y caracterización de los daños.

En el presente artículo se aborda el tema del Monitoreo de Salud Estructural, presentando sus características y alcances. Además, se exponen los avances y perspectivas de dos investigaciones sobre este tema realizados por la Escuela de Ingeniería Civil y Geomática de la Universidad del Valle. Es de anotar que en el país la investigación de este tipo está en una fase incipiente.

\section{MONITOREO DE SALUD ESTRUCTURAL}

El Monitoreo de Salud Estructural (MSE) permite identificar la presencia de daños en la estructura con base en los cambios de sus parámetros estáticos y dinámicos, a través de métodos de modelación analítica y de interpretación de datos. Además, proporciona información sobre el comportamiento de las estructuras, que puede ser comparada con las predicciones del análisis estructural. Los métodos de acústica y ultrasonido son un ejemplo de técnicas de MSE apropiados para detectar daños localizados, pero que no 
tienen en cuenta el comportamiento global de la estructura. De otro lado, los métodos basados en el cambio de frecuencias naturales y modos de vibración, siven para detectar tanto daños locales como globales.

Las técnicas de MSE pueden ser categorizadas en cuatro niveles, según su alcance (Rytter, 1993):

Nivell: Identificación de la presencia del daño.

Nivelll: Identificación de la presencia y ubicación del daño.

Nivel Ill:Identificación de la presencia, ubicación y magnitud del daño.

Nivel N: Predicción de la vida útil de la estructura.

El estado del arte en estas técnicas está en detectar, localizar y cuantificar el daño en las estructuras al comparar su comportamiento antes y después de ser sometidas a solicitaciones de carga como sismos, huracanes o cargas de tráfico; e inclusive predecir el resto de vida útil de la estructura. Estas técnicas simplifican los procedimientos de inspección de daños, ya que permiten al ingeniero hacer la cuantificación sin inspección visual o directa de la estructura.

El equipo empleado en el MSE consiste en transductores (o sensores), acondicionadores de señal, dispositivos para adquisición de datos y soportes físico y lógico (hardware y software) para la interpretación. Los transductores más usados son: acelerómetros (uniaxiales, biaxiales o triaxiales), extensómetros, termocuplas, detectores de resistencia/temperatura, transformadores lineales de voltaje/desplazamiento y detectores de corrosión. Normalmente se instalan los transductores en la estructura y se transmiten las señales por radio, microondas, línea telefónica o fibra óptica hasta una central donde se realiza la interpretación de los datos.
Los datos pueden ser almacenados para un análisis posterior, sin embargo, es posible automatizar la interpretación de los datos y realizar un monitoreo en tiempo real. El monitoreo a tiempo real permite identificar fallas peligrosas y evacuar edificaciones 0 cerrar puentes antes de un colapso.

Las técnicas de MSE cambian totalmente la filosofía del mantenimiento de las estructuras, ya que no es necesaria la presencia de daño visible para empezar la reparación, sino que se previene este daño reparándolo desde sus inicios y así economizando los recursos. Además, la información sobre el comportamiento de las estructuras durante un sismo u otro evento importante, permite evaluar los métodos de diseño existentes, mejorarlos y desarrollar otros nuevos.

EI MSE para estructuras civiles ha ganado sustancial atención por parte de investigadores en los últimos años, como se expone en la recopilación de Doebling y otros (1996). Organizaciones como la Asociación Internacional para Control Estructural (IASC) y la Sociedad Americana de Ingeniería Civil (ASCE), son concientes de la importancia del monitoreo de salud estructural. Estas asociaciones están conduciendo los estudios en diferentes técnicas hacia la formación del Grupo de Operaciones en Problemas de Referencia en Monitoreo de Salud Estructural, proponiendo, a investigadores de todo el mundo, problemas de referencia que son resueltos por diferentes métodos, evaluando así su eficacia. Como ejemplo, en este momento hay un problema propuesto en Internet que consiste en un modelo a escala de un edificio de pórticos metálicos de $2,5 \mathrm{x}$ $2,5 \mathrm{~m}^{2}$ de área y 3,6 $\mathrm{m}$ de altura, al que se le han instalado sensores y se le han provocado daños localizados; los datos obtenidos por los sensores son analizados por los investigadores y éstos deben entregar sus resultados (Johnson y otros, 2000; ver además ). En la Conferencia de Ingeniería Mecánica 2000 de la ASCE, se 
dedicó una sesión a este problema de referencia, donde algunos investigadores expusieron el trabajo hecho hasta el momento (Lus y Betti, 2000; Bernal y Gunes, 2000; Katafygiotis y otros, 2000; Corbin y otros, 2000; Dyke y otros, 2000; Au y otros, 2000). En este evento fue de particular interés el desarrollo de técnicas que consideren los efectos ambientales en la respuesta dinámica de las estructuras, siendo necesario diferenciarlos de los efectos producidos por daños estructurales.

\section{PUENTE “EL HORMIGUERO”}

En la Escuela de Ingeniería Civil y Geomática de la Universidad del Valle se está desarrollando un proyecto de MSE a distancia y en tiempo real. El proyecto consiste en instrumentar y monitorear la estructura del puente "El Hormiguero" (Figura 1), transmitir la señal por radio hasta el Observatorio Sismológico (OSSO) y luego enviarla por cable al Laboratorio de Ingeniería Sísmica y Dinámica Estructural (LINSE), donde se realiza la interpretación de datos. Con este fin, se está implementando un Sistema de Identificación de Daños (SID) de Nivel I. Un aporte importante de este proyecto es demostrar que es posible implementar en este país un sistema de MSE, adecuando tecnología extranjera e implementando equipos construidos en la Universidad.

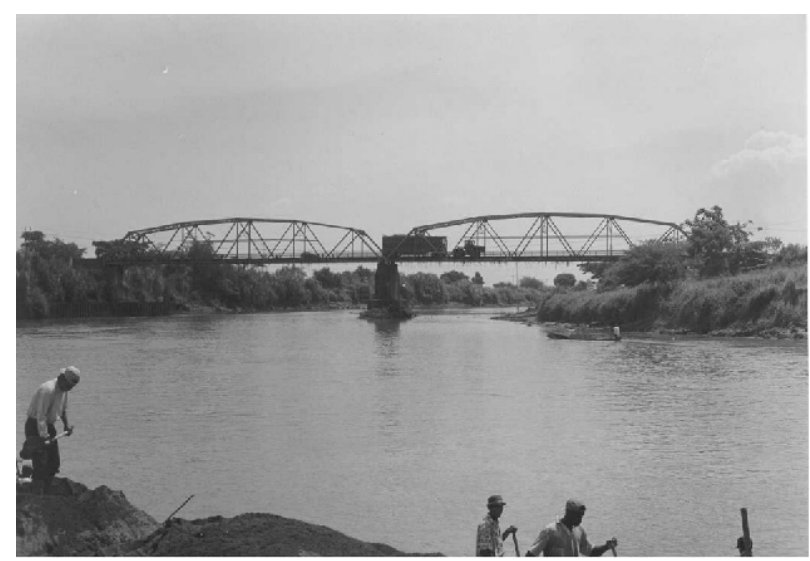

Figura 1. Puente "El Hormiguero"

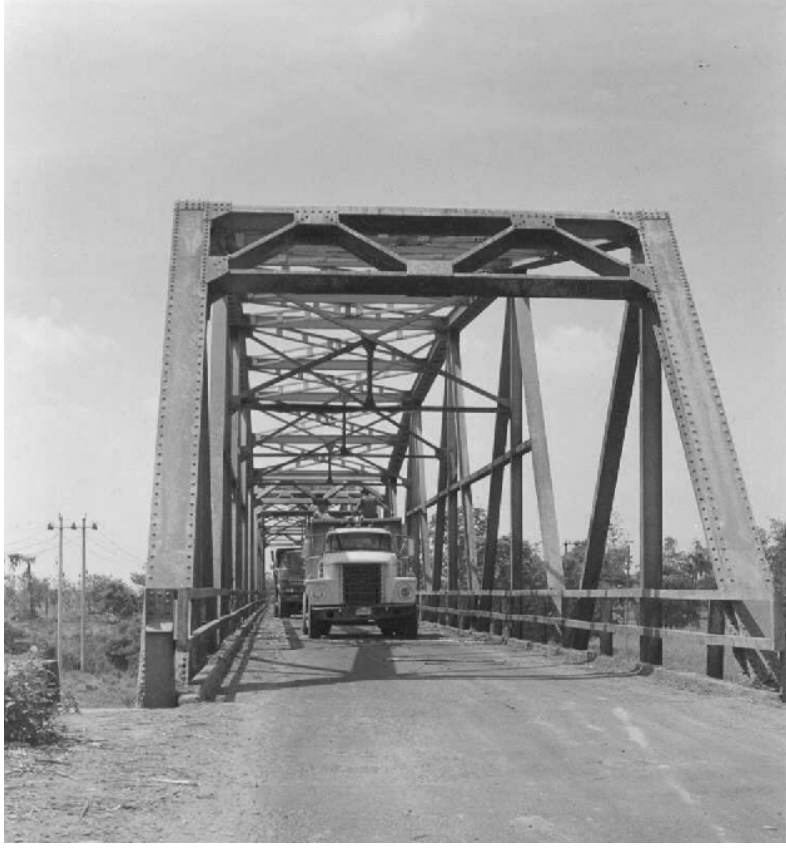

Figura 1. Puente "El Hormiguero"

El puente "El Hormiguero" está localizado en la vía Cali-Puerto Tejada, sobre el río Cauca, a 10 Kilómetros de la Universidad del Valle. La estructura del puente consiste en dos luces de $62 \mathrm{~m}$. simplemente apoyadas. Cada luz consta de dos armaduras metálicas en forma de arco, unidas transversalmente por riostras y diagonales entre las cuerdas superiores y algunos elementos verticales, y por vigas transversales entre las cuerdas inferiores, que soportan las viguetas y el tablero de concreto. En el puente se tiene instalado un acelerómetro sísmico uniaxial WR-731A, un amplificador/filtro WR-P31 y un transmisor de radio FM Monitron TT-2.

La identificación de daños y monitoreo de salud estructural se realiza basado en cambios en las frecuencias naturales y modos de vibración del puente, y mediante comparaciones con un modelo analítico. El modelo analítico fue desarrollado con el programa de análisis estructural por elementos finitos SAP2000 Non Linear y se calibró con respecto a las formas modales y los períodos de vibración obtenidos de mediciones in situ 
(Figura 2). Estas mediciones indican que el pico vertical de aceleraciones del tablero asciende hasta $0.2 \times g$ bajo cargas de tráfico y registran desplazamientos de más de 0.5 centímetros, lo que sugiere que el puente tiene problemas de vibración que deben ser investigados con más detalle. Además, se están introduciendo en el modelo los daños que presente la estructura con el fin de tener una mejor aproximación al comportamiento real.

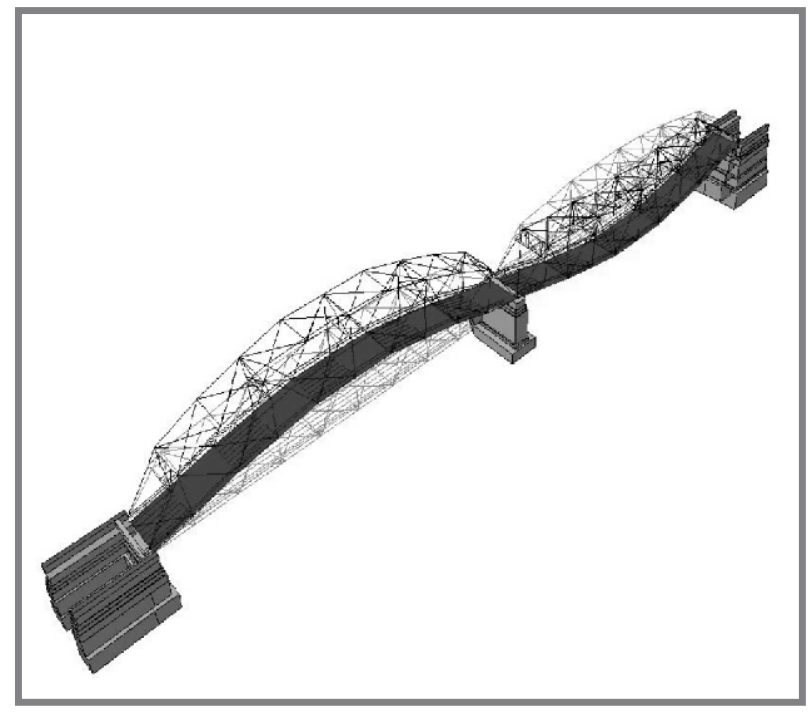

Figura 2. Forma modal del modelo analítico del puente "El Hormiguero"

\section{PUENTE PEREIRA - DOS QUEBRADAS}

El Viaducto César Gaviria Trujillo es la obra de infraestructura vial más importante construida en los últimos años en Colombia (Figura 3). En su construcción se invirtieron aproximadamente 58 millones de dólares, de los cuales 1,5 se destinaron para la compra e instalación de alrededor de 300 sensores para el MSE, que incluyen extensómetros, inclinómetros, medidores de temperatura y medidores de corrosión, distribuidos por la estructura metálica, los pilones y los tirantes. La señal emitida por estos sensores es enviada por un canal serial a una central ubicada cerca al puente, en el extremo de Dos Quebradas.

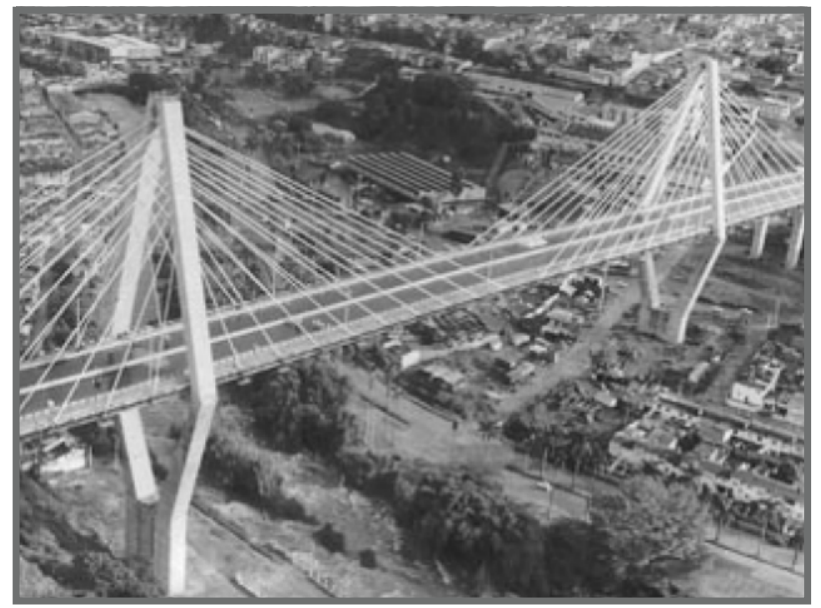

Figura 3. Viaducto Pereira - Dos Quebradas

\section{Descripción General del Sistema de Monitoreo}

El sistema de monitoreo del Viaducto se compone de los siguientes subsistemas:

Subsistema A: Se encarga del monitoreo de los fenómenos lentos tales como los producidos por cambios de temperatura, por el asentamiento de las fundaciones 0 por modificaciones en el comportamiento de la estructura bajo el efecto de la relajación del concreto $O$ de las varias fases de la construcción. Debido a la lenta variación de estos parámetros, la frecuencia de toma de datos es de seis horas con instrumentación automática.

Con este Subsistema se busca obtener información relativa al tablero y los pilones. En cuanto a los pilones se refiere, se mide la temperatura del aire en la vecindad, así como la rotación longitudinal y transversal, las deformaciones en el concreto y el acero, y el movimiento de las juntas del tablero. Para los pilones, se mide la temperatura y la humedad del aire en su vecindad, además de la radiación solar. Adicionalmente, se determina su rotación longitudinal y transversal, y las deformaciones en el concreto. 
Subsistema B: Tiene que ver con el monitoreo de fenómenos rápidos producidos por acciones dinámicas derivadas del tráfico vehicular, el viento y los fenómenos sísmicos. La frecuencia de toma de datos es variable, pues se establecen niveles de umbral a partir de los cuales se inicia la secuencia de adquisición de datos a una tasa que depende de la velocidad de variación de la variable en cuestión.

Este Subsistema proporciona información dinámica del tablero, pilones y tirantes. Para el tablero y los pilones se registra la velocidad y dirección del viento. Adicionalmente, para el tablero se registran la aceleración longitudinal, transversal y vertical, y la deformación de la estructura metálica y de la armadura de acero de la losa de concreto. En los pilones también se registran estas aceleraciones y en los tirantes se registran aceleraciones bidimensionales que permiten definir sus modos de vibración e indirectamente el cálculo de su tensión.

Subsistema C: Está diseñado para detectar la corrosión en el acero de la armadura de concreto. La variación esperada de este parámetro es muy lenta, por esta razón la frecuencia de medición es semestral y se lleva a cabo de forma manual, mediante un dispositivo portátil con el que se busca establecer el potencial de macropar de las barras de la armadura para inferir el nivel de corrosión.

\section{CONVENIO UNIVALLE - UTP}

La Universidad Tecnológica de Pereira (UTP) suscribió en 1998 un convenio con el Instituto Nacional de Vías (INVÍAS) con el fin de realizar el mantenimiento de los equipos y la adquisición de datos del Viaducto Pereira-Dos Quebradas. Sin embargo, el convenio UTP-INVIAS no contemplaba la interpretación de los datos para efectos de monitoreo de la salud estructural del Viadaucto. Por lo tanto, la
Universidad del Valle, a través de la Escuela de Ingeniería Civil y Geomática, suscribió un convenio con la UTP para desarrollar un sistema de Monitoreo de Salud Estructural a distancia y en tiempo real para el Viaducto Pereira-Dos Quebradas.

El convenio UTP-Univalle tiene los siguientes objetivos específicos: monitoreo y diagnóstico del estado de la salud estructural del Viaducto, y desarrollo de un sistema de diagnóstico del estado de salud estructural y de alerta de fallas en la estructura. Este sistema se desarrollará en las siguientes dos fases:

Fase I: a) Modelación analítica de la estructura. b) Correlación entre factores ambientales y parámetros estáticos y dinámicos. c) Diseño de un software para alerta de fallas repentinas en elementos estructurales y su implementación en la estación de monitoreo del Viaducto en Dos Quebradas.

Fase II: a) Sistema de Identificación de Daños de Nivel II (identificación del daño y su ubicación) en tiempo real, con base en los parámetros dinámicos y estáticos. b) Sistema de Identificación de Daños de Nivel III (identificación del daño, su ubicación y magnitud) en tiempo real.

\section{CONCLUSIONES}

Las técnicas de Monitoreo de Salud Estructural son una buena solución al problema de la detección de daños por métodos no destructivos, basados en los cambios de los parámetros estáticos y dinámicos de la estructura. La inversión adicional en equipos de monitoreo es recompensada con el ahorro en inspecciones y la efectividad en la prevención de desastres. Tal como se ha mostrado con el puente El Hormiguero, es posible implementar sistemas de MSE en Colombia a bajo costo y desarrollando tecnología propia. Para el caso del Viaducto Pereira-Dos Quebradas, se tiene 
un laboratorio a escala real que abre las puertas al desarrollo de proyectos de punta en investigación sobre detección de daño.

\section{RECOMENDACIONES}

En este aparte los autores exponen algunas recomendaciones presentadas en la recopilación de Doebling y otros (1996), para nuevas investigaciones en Monitoreo de Salud Estructural:

- Desarrollar un modelo analítico previo al monitoreo.

- Usar métodos no lineales de análisis.

- Identificar el efecto de los factores ambientales.

- Abordar los problemas propuestos por la IASC y la ASCE.

- Trabajar con estructuras reales.

\section{BIBLIOGRAFÍA}

DOEBLING, S. W., FARRAR, C. R., PRIME, M. B., Y SHEVITZ, D. W. "Damage Identification and Health Monitoring of Structural and Mechanical Systems From Changes in Their Vibration Characteristics: A Literature Review". Los Alamos National Laboratory Report LA13070-MS. 1996.

JOHNSON, E.A., LAM, H.F., KATAFYGIOTIS, L., Y BECK, J. (2000). "A Benchmark Problem for Structural Health Monitoring and Damage Detection". Proceedings of the 14th ASCE Engineering Mechanics Conference. Austin, Texas: May 2124, 2000.

H. LUS Y R. BETTI. "Damage Identification in Linear Structural Systems". Proceedings of the 14 th ASCE Engineering Mechanics Conference. Austin, Texas: May 2124, 2000.

BERNAL D. y GUNES B. "Observer/Kalman and Subspace Identification of the UBC Benchmark Structural Model". Proceedings of the 14th ASCE Engineering Mechanics Conference. Austin, Texas: May 21-24, 2000.

KATAFYGIOTIS, L.S. , Lam, H.F. y Mickleborough,
N. "Application of a Statistical Approach on as Benchmark Damage Detection Problem". Proceedings of the 14th ASCE Engineering Mechanics Conference. Austin, Texas: May 21-24, 2000.

CORBIN, M., HERA, A., y HOU, Z. "Locating Damage Regions Using Wavelet Approach". Proceedings of the 14th ASCE Engineering Mechanics Conference. Austin, Texas: May 21 24, 2000.

DYKE, S.J., CAICEDO, J.M., y JOHNSON, E.A. "Monitoring of a Benchmark Structure for Damage Detection". Proceedings of the 14th ASCE Engineering Mechanics Conference. Austin, Texas: May 21-24, 2000.

AU S. K., YUEN K.V., y BECK J. "Two-Stage System Identification Results for Benchmark Structure". Proceedings of the 14th ASCE Engineering Mechanics Conference. Austin, Texas: May 21 24, 2000.

CAICEDO J. M., DYKE S. J., y JOHNSON E. "Health Monitoring Based on Component Transfer Functions". Proceedings of the 14th ASCE Engineering Mechanics Conference. Austin, Texas: May 21-24, 2000.

FARRAR, C.R. y JAMES, G.H. III. "System Identification from Ambient Vibration Measurements on a Bridge" Journal of Sound and Vibration. 205(1), pp. 118.1997.

CHANG, F.-K. "Structural Health Monitoring". Proceedings of the 2nd International Workshop on Structural Health Monitoring. Stanford University, September 810, 1999. Technomic Publishing Co., Lancaster, PA. 1999.

RYTTER, A. "Vibration Based Inspection of Civil Engineering Structures". Dissertation, Department of Building Technology and Structural Engineering. Aalborg University, Denmark. 1993.

http://ohm.utp.edu.co/viaducto

http://wusceel.cive.wustl.edu/asce.shm/ 